MATEC Web of Conferences 32, 02002 (2015)

DOI: $10.1051 /$ matecconf $/ 20153202002$

(O) Owned by the authors, published by EDP Sciences, 2015

\title{
Holographic vector-wave femtosecond laser processing for fabrication of orientation-controlled nanostructures
}

\author{
Satoshi Hasegawa ${ }^{a}$ and Yoshio Hayasaki \\ Center for Optical Research and Education (CORE), Utsunomiya University, 7-1-2 Yoto, Utsunomiya, 321-8585, Japan
}

\begin{abstract}
We demonstrated holographic vector-wave femtosecond laser processing system based on a holographic manipulation of the light including amplitude, phase and polarization. The system performs a high-throughput and flexible fabrication of orientation-controlled nanostructures applied to material surface modification for control of a tribology, wettability, light reflectance and retardance. In this experiment, a polarization characteristic of the laser processing system was experimentally verified. In addition, parallel fabrication of orientation-controlled nanostructures using multifocal vector beams was performed.
\end{abstract}

\section{Introduction}

A spatial light modulator (SLM) displaying a dynamic computer-generated hologram (CGH) has been used for parallel femtosecond laser processing [1] based on an array of spots with a variable patterning. It was called holographic femtosecond laser processing $[2,3]$, and has the advantages of high-throughput pulsed irradiation and high energy-use efficiency of the pulse. The holographic femtosecond laser processing has been widely used in many applications, for example, optical waveguide fabrication [4], fabrication of volume phase gratings in polymers [5], surface structuring of silicon [6], twophoton polymerization [7], two-photon microscopy [8] and optical tweezers [9]. These applications have been mainly performed based on a holographic control of spatial phase of the light.

For opening up a wide variety of new applications, enhancing the potential ability of femtosecond laser processing is essential. Exploring the nature of the light as a vector wave, including amplitude, phase and polarization is effective way to enhance the ability. Control of the polarization state of femtosecond laser light has played a significant role in various applications because it provides efficient control of light-matter interactions. In particular, a light field with cylindrical symmetry of linear polarization, called a vector beam, has attracted attention due to the novel properties it offers, such as selective excitation of an anisotropic materials distributed randomly in three-dimensional (3D) space [10], observation of the 3D orientation of a biological cell [11], effective laser cutting [12], and fabrication of nanostructure [13] aligned perpendicularly to the polarization direction of the light due to an interaction between a femtosecond laser light and a surface plasmon wave. As mentioned above, previous researches have been based on an individual control of degrees of freedom in the light including an amplitude, phase and polarization. However, there have been few reports on the full control of that.

In this study, we have demonstrated femtosecond laser processing with full control of the light using CGHs. It was called holographic vector wave femtosecond laser processing $[14,15]$. The novel beam-control technique provides quantitative and qualitative change to femtosecond laser processing, such as a high-throughput and flexible fabrication of orientation-controlled nanostructures applied to material surface modification for control of a tribology, wettability, light reflectance and retardance. In the experiment, a characteristic of polarization modulation in the laser processing system was experimentally measured. Secondary, a typical case of laser processing with a single vector beam focused by an objective lens with a numerical aperture (NA) of 0.60 was performed. Finally, parallel fabrication of spatially complex nanostructures using multifocal vector beams was demonstrated. An orientation of fabricated nanostructure agreed well with the designed polarization state. In Sec. 2, we describe the principle of the full control of phase distribution and polarization state of light using two SLMs. In Sec. 3, we present our experimental setup. In Sec. 4, we show the experimental results. We give some concluding remarks in Sec. 5.

\section{Principle}

The optical setup mainly consists of a half-wave plate (HWP), a quarter-wave plate (QWP), and a pair of SLMs. When the Jones matrixes of these optical elements are defined as $H, Q$, and $S$, respectively, the Jones vector of the output beam from the setup, $\mathbf{E}_{\text {out }}$, is expressed as

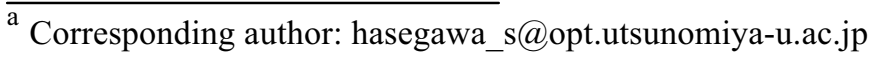




$$
\begin{aligned}
\mathbf{E}_{\text {out }} & =Q_{\frac{\pi}{4}} S_{\beta} H_{\frac{\pi}{8}} S_{\alpha} \mathbf{E}_{\text {in }} \\
& =\frac{1}{2}\left[\begin{array}{cc}
-i \exp [i(\alpha+\beta)]+\exp (i \alpha) & i \exp (i \beta)+1 \\
-\exp [i(\alpha+\beta)]+i \exp (i \alpha) & \exp (i \beta)+i
\end{array}\right] \mathbf{E}_{\text {in }} \\
& =\frac{\exp (i \alpha)}{2}\left[\begin{array}{c}
-i \cos \beta+\sin \beta+1 \\
-\cos \beta-i \sin \beta+i
\end{array}\right]
\end{aligned}
$$

where $\mathbf{E}_{\text {in }}=(1,0)^{\mathrm{T}}$ is the Jones vector of an input beam with linear polarization, the subscripts on $H$ and $Q$ are the azimuthal angles at the respective wave plates, and the subscripts $\alpha$ and $\beta$ are the phases applied to the pair of SLMs, respectively. As shown in Eq. (1), the phase distribution and polarization state of the output beam are independently controlled by the phases $\alpha$ and $\beta$, respectively. These controls correspond to designing the configuration of array of spots and the polarization of the each spot, respectively. Figure 1 shows a polarization direction of output beam versus phase $\beta$ obtained by Eq. (1). In the figure, a slope and y-intercept are normally set to $1 / 2$ and $-\pi / 4$, respectively.

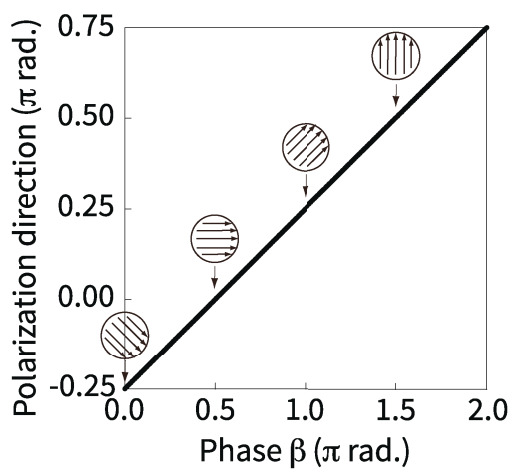

Figure 1. Polarization direction of output beam versus phase $\beta$ applied to SLM.

\section{Experimental setup}

Figure 2 shows a holographic vector-wave femtosecond laser processing system. It is mainly composed of an amplified femtosecond laser system (Coherent, Micra and Legend Elite), liquid-crystal-on-silicon SLMs (LCOSSLMs; Hamamatsu Photonics, X10468-02), laser processing optics, and a personal computer (PC). The incident femtosecond pulse had a center wavelength of $800 \mathrm{~nm}$, a spectral width of $25 \mathrm{~nm}$ FWHM, a pulse width of $40 \mathrm{fs}$, a repetition frequency of $1 \mathrm{kHz}$, and linear polarization with a p-component. The pulse was radiated onto the first SLM (SLM1), which displayed CGH1 for applying a pure phase delay to the p-component, that is, phase modulation. The HWP was arranged with an azimuthal angle of $\pi / 8$ to rotate the linear polarization by $\pi / 4$. The pulse was also radiated onto the second SLM (SLM2), which displayed $\mathrm{CGH} 2$ for applying a phase delay between the $\mathrm{p}$ - and s-components, that is, polarization modulation. SLM2 was located at the image plane of SLM1. The circular or elliptical polarization reflected from SLM2 was converted to linear polarization using a QWP set to an azimuthal angle of $\pi / 4$.
Consequently, the reconstructed spot array with a desired polarization distribution was obtained at plane $\mathrm{P}$ and was directed to the laser processing optics, containing a $40 \times$ objective lens $(\mathrm{OL})$ with $\mathrm{NA}=0.60$. To observe laser processing of the sample, the sample was illuminated with a halogen lamp (HL), and a charge coupled device (CCD) image sensor captured images of the sample via a dichroic mirror (DM) and an infrared (IR) cut filter. The sample was a super-white crown glass (Schott, B270). The structure fabricated by the laser irradiation was observed using a transmitted optical microscope (Olympus, BX-51) and a scanning electron microscope (SEM; Hitachi, S-4500).

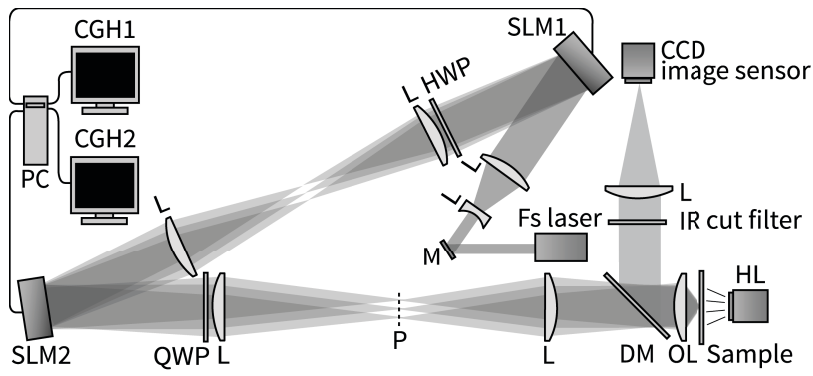

Figure 2. Holographic vector-wave femtosecond laser processing system.

\section{Experimental results}

Firstly, in order to verify a characteristic of polarization modulation in the optical setup, a light intensity of the output beam was measured through a linear polarizer arranged at the front of a photodetector. An azimuthal

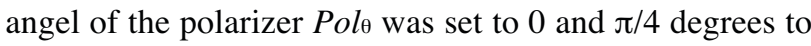
determine a departure point and an orbital direction on the Poincare sphere describing the polarization state. Figure 3 shows a transmitted light intensity $I_{\mathrm{t}}$ through the polarizer as a function of phase $\beta$ applied to SLM2 when the azimuthal angles of HWP and QWP were set to $\pi / 8$ and $\pi / 4$. A filled circle and open circle indicate the result when $P o l_{\theta}$ was set to 0 and $\pi / 4$ degrees, respectively. A solid line means a theoretical value expressed as follows.

$$
I_{t}=A_{t}^{2}=\cos ^{2}\left(\operatorname{Pol}_{\theta}-\operatorname{Beam}_{\theta}\right)=\cos ^{2}\left[\operatorname{Pol}_{\theta}-(a \beta+b)\right],
$$

where $A_{\mathrm{t}}$ is an amplitude of transmitted light and Beame is a polarization direction of the output beam. $a$ and $b$ mean

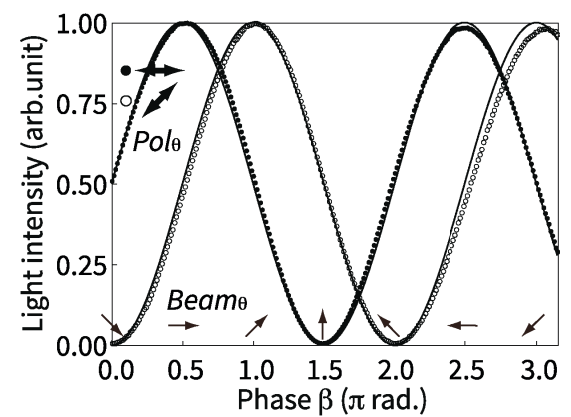

Figure 3. Transmitted light intensity of output beam through a polarizer versus phase $\beta$. 
a slope and y-intercept of the line shown in Fig. 1, respectively. The experiment agreed well with the theoretical value. A contrast ratio of the experiment value was approximately 150:1.

Figure 4 shows a typical case of a laser processing using a single vector beam. Figures 4 (a) to 4(d) show the case of radial polarization beam. Figures 4(a) and 4(b) show CGHs for the phase and polarization modulation, respectively. The gray scale corresponds to the phase from 0 to $2 \pi$. Figures $4(\mathrm{c})$ is the optical reconstruction captured at plane $\mathrm{P}$ in Fig. 2. In the figure, the white arrows mean the polarization direction. The reconstruction had a doughnut-shaped intensity at focus because each focal spot had a phase and polarization singularity at the beam center. Figure 4(d) shows SEM images from top view of structure fabricated by the vector beam. Processing was performed by the pulse energy of $270 \mathrm{~nJ}$ and pulse shot number of 10000 . A pitch of fabricated nanostructure was approximately 180 $\mathrm{nm}$. An orientation of nanostructure was perpendicularly arraigned to the polarization state of vector beam. Figures $4(\mathrm{e})$ to $4(\mathrm{~h})$ show the case of azimuth polarization beam. Figures 4(i) to 4(1) show the case of windmill polarization beam. Interestingly, vortical nanostructure was successfully fabricated. From each SEM image, three types of spatially complex nanostructure were fabricated on the sidewall of the hole.

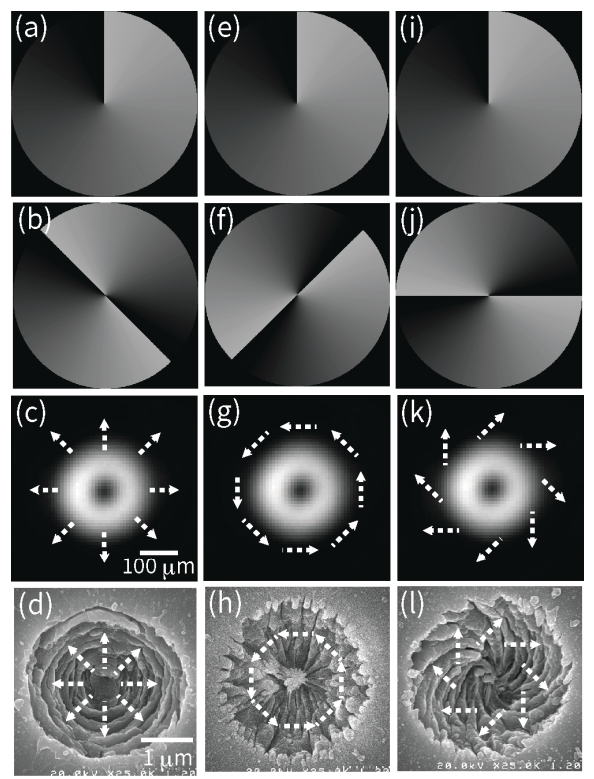

Figure 4. Typical cases of laser processing using a single vector beam. (a)-(d) In the case of radial polarization beam. (a)-(c) The $\mathrm{CGH}$ for the phase and polarization modulation, and its optical reconstruction, respectively. (d) SEM images from a top view of fabricated structure. In the case of (e)-(h) azimuth polarization beam and (i)-(1) windmill polarization beam, respectively.

Figures 5(a) and 5(b) show CGHs for modulating the phase distribution and the polarization state, respectively. Figure 5(c) shows the optical reconstruction of multifocal radial and azimuth beams. Figures 5(d) and 5(e) show the reconstructions captured through a linear polarizer with azimuthal angles of $0 \pi$ and $\pi / 2$ radians,
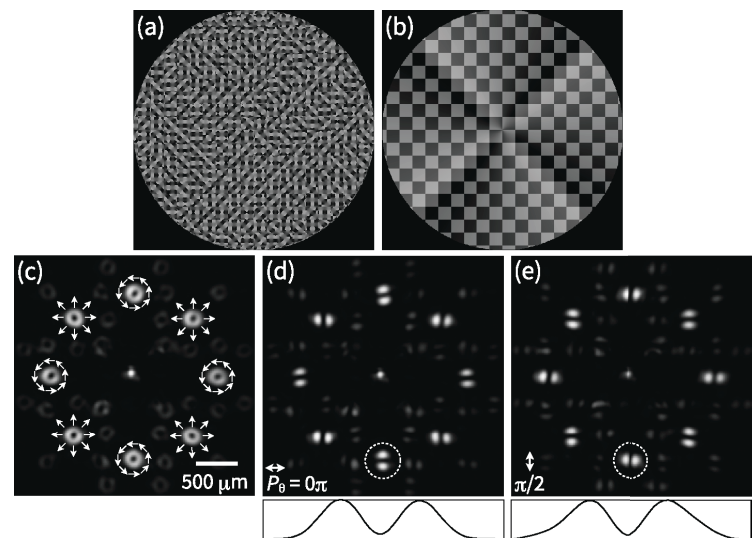

Figure 5. (a), (b) CGH for modulating the phase distribution and the polarization state, respectively. (c) Optical reconstruction of multifocal radial and azimuth beams. (d), (e) The reconstructions captured through a linear polarizer with azimuthal angles of $0 \pi$ and $\pi / 2$ radians, respectively.

respectively. The profiles mean the intensity distribution corresponding to the focal spot inside the dashed circle.

In order to confirm the polarization states of the focal spot at the sample plane, laser processing was performed. In the processing result, the polarization state was analysed from the orientations of the fabricated nanostructures. Figures 6(a) and 6(b) show SEM images of the structure fabricated using the reconstruction (Fig. $5(c))$ and its magnified images at each location when the pulse shot number was set to 1 and 100, respectively. The irradiation pulse energy was fixed to $4.7 \mu \mathrm{J}$ in each case. From the results, the orientation of fabricated nanostructures was agreed well with the polarization state of each beam. Two types of orientation-controlled nanostructures with a pitch of $150 \mathrm{~nm}$ were simultaneously fabricated.

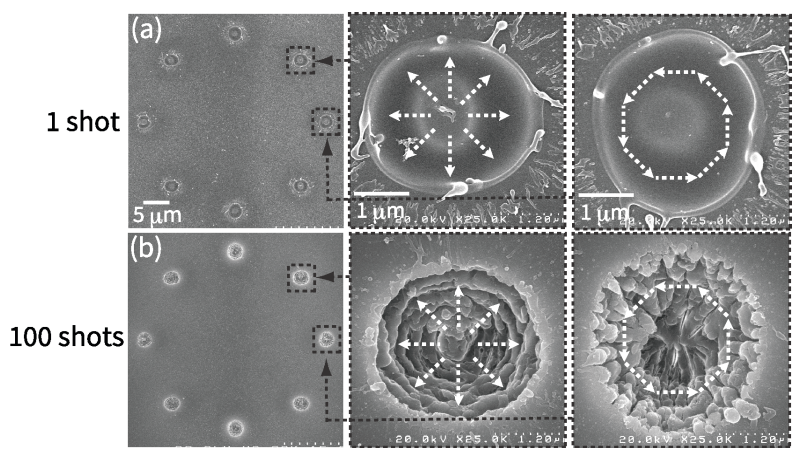

Figure 6. SEM images of the structure fabricated using the multifocal radial and azimuth beams with the pulse shot number of (a) 1 and (b) 100, respectively.

\section{Conclusion}

We demonstrated holographic femtosecond laser processing with full control of phase distributions and polarization states of light. A characteristic of polarization modulation in the laser processing system was experimentally measured. In the system, parallel fabrication of orientation-controlled nanostructures using 
multifocal vector beams was performed. An orientation of fabricated nanostructure agreed well with the polarization state of designed vector beam. Our proposed system useful for a high-throughput and flexible fabrication of orientation-controlled nanostructure applied to material surface modification for control of a tribology, wettability, light reflectance and retardance.

\section{References}

1. Y. Kuroiwa, N. Takeshima, Y. Narita, S. Tanaka, and K. Hirao, Opt. Express 12, 1908 (2004).

2. Y. Hayasaki, T. Sugimoto, A. Takita, and N. Nishida, Appl. Phys. Lett. 87, 031101 (2005).

3. N. Sanner, N. Huot, E. Audouard, C. Larat, J. P. Huignard, and B. Loiseaux, Opt. Lett. 30, 1479 (2005).

4. M. Sakakura, T. Sawano, Y. Shimotsuma, K. Miura, and K. Hirao, Jpn. J. Appl. Phys. 48, 126507 (2009).

5. D. Liu, Z. Kuang, W. Perrie, P. J. Scully, A. Baum, S. P. Edwardson, E. Fearon, G. Dearden, and K. G. Watkins, Appl. Phys. B 101, 817 (2010).

6. H. Imamoto, S. Kanehira, X. Wang, K. Kametani, M. Sakakura, Y. Shimotsuma, K. Miura, and K. Hirao, Opt. Lett. 36, 1176 (2011).

7. S. D. Gittard, A. Nguyen, K. Obata, A. Koroleva, R. J. Narayan, and B. N. Chichkov, Biomed. Opt. Express 2, 3167 (2011).

8. V. R. Daria, C. Stricker, R. Bowman, S. Redman and, H. A. Bachor, Appl. Phys. Lett. 95, 093701 (2009).

9. M. Antkowiak, M. L. Torres-Mapa, F. Gunn-Moore, and K. Dholakia, J. Biophotonics 3, 696 (2010).

10. X. Li, T. H. Lan, C. H. Tien, and M. Gu, Nat. Commun. 3, 998 (2012).

11. K. Yoshiki, M. Hashimoto, and T. Araki, Jpn. J. Appl. Phys. 44, L1066 (2005).

12. M. Meier, V. Romano, and T. Feurer, Appl. Phys., A Mater. Sci. Process. 86, 329 (2007).

13. Y. Shimotsuma, P. G. Kazansky, J. Qiu, and K. Hirao, Phys. Rev. Lett. 91, 247405 (2003).

14. S. Hasegawa and Y. Hayasaki, Opt. Express 21, 12987 (2013).

15. S. Hasegawa and Y. Hayasaki, Int. J. Optomechatronics 8, 73 (2014). 